\title{
Recent Advances in Simple
} Preparation of 3D Graphene Aerogels Based on 2D Graphene Materials

\author{
Meichun Ding ${ }^{1,2}$ and Chenwei $L i^{*, 1,2}$ \\ ${ }^{1}$ School of Chemistry and Pharmaceutical Engineering, Shandong First Medical University and Shandong Academy of Medical \\ Sciences, Jinan, China, ${ }^{2}$ Medical Science and Technology Innovation Center, Shandong First Medical University and Shandong \\ Academy of Medical Sciences, Jinan, China
}

Recently, 3D graphene aerogels (3GAs) with high electrical conductivity, excellent mechanical properties, and fast mass and electron transport have attracted increasing attention and shown wide applications (such as flexible electronics devices, sensors, absorbents, catalysis, energy storage devices, solar steam generation devices, and so on). The drying process becomes an important factor limiting the large-scale preparation of 3GAs. Therefore, how to simplify the preparation process plays an important role in the large-scale application of 3GAs. In this study, we summarize the recent progresses of 3GAs by different drying methods and focus on the effect of robust graphene network on the simple preparation of 3GAs. Besides, the design and synthesis strategies of 3GAs with robust graphene network structures have been systematically discussed. Finally, the

OPEN ACCESS

Edited by:

Florian Levet,

University of Cagliari, Italy

Reviewed by:

Xiehong Cao,

Zhejiang University of Technology,

China

*Correspondence:

Chenwei $\mathrm{Li}$

lichenwei@iccas.ac.cn

Specialty section:

This article was submitted to Nanoscience,

a section of the journal

Frontiers in Chemistry

Received: 15 November 2021 Accepted: 03 January 2022

Published: 26 January 2022

Citation:

Ding M and Li C (2022) Recent Advances in Simple Preparation of $3 D$ Graphene Aerogels Based on 2D

Graphene Materials.

Front. Chem. 10:815463.

doi: $10.3389 /$ fchem.2022.815463 emerging challenges and prospective for developing simple preparation and functionalization of 3GAs were outlined. It is expected that our study will lay a foundation for large-scale preparation and application of 3GAs and inspire more new ideas in this field.

Keywords: graphene, aerogels, 3D porous materials, drying method, elasticity

\section{INTRODUCTION}

The aerogel is a kind of three-dimensional porous material prepared from a precursor by a sol-gel process and a suitable drying method. The concept of "aerogel" was first presented by S. Kistler in the 1930s when he successfully obtained a porous $\mathrm{SiO}_{2}$ material of the same size as the original by using a supercritical drying method (Kistler, 1931a). Since then, inorganic aerogels $\left(\mathrm{SiO}_{2}\right.$ aerogels, $\mathrm{TiO}_{2}$ aerogels, $\mathrm{Al}_{2} \mathrm{O}_{3}$ aerogels, and so on), organic aerogels (resorcinol-formaldehyde aerogels, melamine-formaldehyde aerogels, and so on), carbon-based aerogels, and so on have been widely used in many fields for their unique properties (low density, high porosity, high surface area, and low thermal conductivity). Since the 2010 Nobel Prize in Physics honored "the new 2D graphene material", the atomic-thick graphene has attracted significant attention because of its unprecedented properties such as high electrical conductivity, excellent mechanical properties, high thermal conductivity, and high surface area (Novoselov et al., 2005). Thanks to the strong interactions between 2D graphene sheets, a tight face-to-face stack structure is formed. Therefore, 2D graphene materials are ideal building blocks for the synthesis of $3 \mathrm{D}$ graphene aerogels (3GAs) compared to 1D and 0D materials (Qiu et al., 2018). Until now, great efforts have been made to prepare a new family of 3GAs with extraordinary performances such as high electrical conductivity, excellent mechanical properties, and fast mass and electron transport, which 
conventional porous materials cannot offer (Lu et al., 2016; Huo et al., 2019; Li et al., 2019). 3GAs can be used in a range of applications including flexible electronics devices (Qiu et al., 2014), sensors (Qiu et al., 2016; Kong et al., 2021), absorbents (Bi et al., 2012), catalysis (Liang et al., 2018), energy storage devices (Jiang et al., 2017; Qu et al., 2020), and solar steam generation devices (Zhang et al., 2017; Yang et al., 2018; Huo et al., 2019; Li et al., 2021).

3GAs could be fabricated by using various methods such as the template-mediated assembly (Li et al., 2014), chemical vapor deposition (CVD) (Bi et al., 2015), 3D printing (Zhu et al., 2015), and self-assembly (Lu et al., 2016). Graphene oxide (GO) is a kind of functionalized graphene having an oxidation group such as an epoxy group and a hydroxyl group, which can be conveniently obtained by peeling off graphite oxide into a layered sheet (Zhao et al., 2015). Compared to other methods, the GObased self-assembly method is the most promising method for large-scale preparation of 3GAs (Nardecchia et al., 2013). To form 3GAs, 2D GO sheets are assembled by dispersing GO in a solution. In the GO solution, the GO sheets are uniformly dispersed because of a balance between the electrostatic repulsion and van der Waals interaction (Cao et al., 2014). Gelation occurs by adding a crosslinking agent, sonicating the GO solution, changing the $\mathrm{pH}$ of the GO solution, and chemical or hydrothermal reduction to break the balance (Li and Shi, 2012). During the gelation process, the graphene sheets are chemically or physically assembled to form the graphene network structure. After gelation, graphene sheets are physically or chemically linked to form network structures; followed by the drying process, 3GAs are obtained (Nardecchia et al., 2013). In order to remove the water of graphene hydrogels, special drying techniques (such as freeze drying or supercritical drying) are often required due to the low solid content of graphene hydrogels (Ma and Chen, 2015). Due to the improvement of the preparation method, the cost of raw materials such as GO is continuously reduced (Kong et al., 2019). The drying process becomes an important factor limiting the large-scale application of graphene porous materials. Therefore, how to simplify the drying process plays an important role in the large-scale application of graphene porous materials. In this minireview, the drying methods of 3GAs in recent years are summarized, and the influencing factors of mechanical performances of 3GAs are reviewed and prospected, in order to promote the large-scale application of 3GAs and inspire new ideas for the development of the simple preparation and functionalization of 3GAs.

\section{Drying Methods}

\section{Supercritical Drying Method}

Due to the low solid content of graphene hydrogels, the graphene network collapses by the high capillary force of solvent evaporation when using the conventional drying methods ( $\mathrm{Li}$ et al., 2016). In order to reduce the damage of the network structure by capillary force during the drying process, special drying methods (supercritical drying and freeze drying) are required. Supercritical drying is the most common method for preparing $\mathrm{SiO}_{2}$ aerogels (Zhang and Scherer, 2017). During the

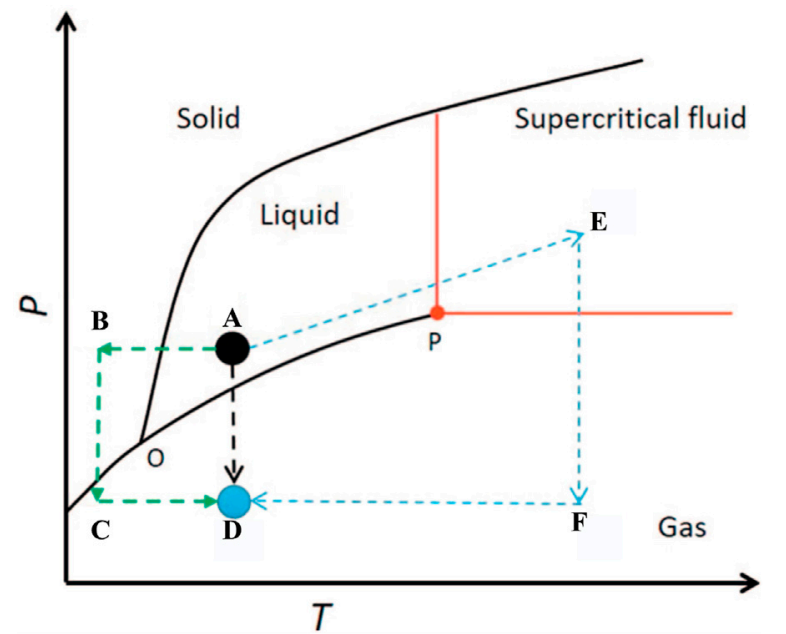

FIGURE 1 | Single-phase phase diagram (Kistler, 1931b).

supercritical drying, the solvent (A spot in Figure 1) becomes a supercritical fluid (E spot in Figure 1) without a liquid-gas interface by adjusting the temperature and pressure, resulting into no capillary pressure in the network structures. When the temperature is kept above the critical temperature, the supercritical fluid can be removed ( $F$ spot in Figure 1) by depressurization to obtain aerogels ( $\mathrm{D}$ spot in Figure 1) (Kistler, 1931b). The $\mathrm{SiO}_{2}$ aerogels dried by supercritical drying basically maintains the original network structure, and the amount of shrinkage is small (Zhang and Scherer, 2017). Compared with the $\mathrm{SiO}_{2}$ aerogel network composed of Si-O covalent bonds, the 3GA is constructed by graphene sheets through $\pi-\pi$ interaction, and the graphene sheets are prone to slip during supercritical drying, resulting in a larger volume shrinkage and a larger density (Zhang et al., 2011; Sui et al., 2012; Huang et al., 2013a). Therefore, the supercritical drying method is not suitable for preparing low density and big-scale 3GAs. Moreover, the supercritical drying method involves the use of high pressure and a long-time process, resulting in high preparation costs.

\section{Freeze Drying Method}

To eliminate capillary forces during the drying process, freeze drying is another option (Zhang and Scherer, 2017). The solvent in the wet gel is first replaced with water. Then, hydrogel (A spot in Figure 1) is first frozen (B spot in Figure 1) under low temperature. Finally, ice is sublimated into gas (C spot in Figure 1) under vacuum without going through the liquid state, thus keeping the graphene skeleton intact (D spot in Figure 1). For the $\mathrm{SiO}_{2}$ gel with a small pore size (tens of nanometer), the volume expansion of water after freezing may destroy the network structure, resulting in the decrease in porosity (as low as $80 \%$ ) and the specific surface area (by half compared to supercritical drying) and cracking of the sample (Maleki, 2016). Due to the large pore sizes (several micrometers to several tens of micrometers), graphene hydrogels are more 
suitable for preparation using freeze-drying than $\mathrm{SiO}_{2}$ hydrogels. The 3 GAs prepared by freeze drying have a lower density (as low as $0.16 \mathrm{mg} \mathrm{cm}^{-3}$ ), and the network structure can be controlled by ice crystals (Hu et al., 2013; Sun et al., 2013). However, the freezing of water may destroy and extrude the microstructure of the graphene network structure, resulting in the low specific surface area (Scherer, 1993). Moreover, freeze drying needs low temperature, high vacuum, and a long-time drying, resulting in high energy consumption and high preparation costs (Gallé, 2001).

\section{Vacuum Drying Method}

The supercritical drying and freeze drying require the use of low vacuum, low temperature, or high pressure, resulting in the high cost of preparation of 3GAs, which will be challenging for largescale production of 3GAs. Compared with supercritical drying and freeze drying, vacuum drying is a simple and time-saving process without requiring solvent exchange and expensive instruments for high pressure and/or temperature (Wang et al., 2013; Li et al., 2016). However, when the graphene gels are dried in a vacuum environment, the $3 \mathrm{D}$ network structures severely collapse, resulting in a sharp increase in the density. Therefore, vacuum drying is an appropriate way to fabricate the assembled structure of the 3D dense graphene network. Therefore, vacuum drying is a suitable method to fabricate the 3D dense graphene network assembly structure for highvolumetric/gravimetric energy density batteries (Li et al., 2018a).

\section{Air Drying Method}

Air drying is a simple and low-cost technique for drying $\mathrm{SiO}_{2}$ gels under ambient pressure conditions and is more suitable for largescale production of aerogels than supercritical drying and freeze drying (Maleki et al., 2015). However, according to the Young-Laplace equation $(\triangle P=2 \gamma \cos \theta / r$, where $\triangle P$ is the capillary pressure, $\gamma$ is the surface tension of the solvent, $\theta$ is the contact angle of the air/fluid interface with the pore wall, and $r$ is the pore size), the interface between liquid and air in a pore structure creates large capillary pressure during the air drying process due to the small pore size (tens of nanometer) (Smith et al., 1995). Because of the capillary pressure, the skeleton of the $\mathrm{SiO}_{2}$ gel gradually shrinks and cracks during the air-drying process. In order to reduce the capillary force, the solvent in the wet gel is first replaced with a solvent having a small surface tension (Zhang et al., 2011). Moreover, the skeleton of the $\mathrm{SiO}_{2}$ gel generally requires chemical treatment with a non-polar group to avoid further condensation of adjacent surface functional groups on the skeleton after compression by capillary stresses during the air-drying process (Maleki, 2016). For 3GAs, the pore structure is subjected to less capillary forces than $\mathrm{SiO}_{2}$ aerogels due to large pore sizes (several micrometers to several tens of micrometers). Therefore, 3GAs are more suitable for large-scale preparation using the air-drying method.

Recently, some researchers prepared the 3GAs by using the air-drying method. We fabricated robust and elastic $(>80 \%$ strain) 3GAs through in situ polymerization of polyacrylamide to reinforce the graphene network (Figure 2A) (Li et al., 2016). No supercritical drying or freeze drying was required to prepare the 3GAs. The robust graphene network could resist the capillary force caused by solvent volatilization during vacuum drying and air drying and maintains low density $\left(7.0-8.6 \mathrm{mg} \mathrm{cm}^{-3}\right)$. Zhang et al. prepared the 3GAs by using GO liquid crystal-stabilized bubbles as a template (Figure 2B) (Zhang et al., 2019). The 3GAs with robust and uniform pore structures could be prepared using the air-drying method. The 3GAs can rapidly recover from the compression of $99 \%$ strain and maintain its original shape after 100,0000 compressive cycles at 70\% strain. Yang et al. developed a method to prepare air-dryable graphene hydrogels by using ice crystals and air bubbles as templates (Figure 2C) (Yang et al., 2019). The superelastic 3GAs are obtained by a wet-press method based on the air-dryable graphene hydrogels. The 3GAs showed outstanding compressive stress $(\sim 47 \mathrm{MPa})$ and superelasticity (can recover from the compression of $97 \%$ strain). Therefore, 3GAs that can be dried by air drying require robust graphene network structures to resist capillary forces. In general, these factors such as GO sheet sizes, interactions between graphene sheets, and graphene network structures are related to the mechanical performances of 3GAs. How to adjust these factors is critical to building robust graphene networks.

\section{Influencing Factors of the Mechanical Performances of 3GAs GO Sheet Sizes}

As the basic unit of a graphene network structure, the properties of the GO sheets affect the mechanical performance of the 3GAs. Based on the 3GAs prepared by using large GO sheets $(\sim 20 \mu \mathrm{m})$ (Figure 3A) and small GO plates $(\sim 2 \mu \mathrm{m})$ (Figure 3B), Ni et al. studied the effect of GO sheet sizes on the mechanical performance of 3GAs (Ni et al., 2015). The 3GAs prepared by large and small GO sheets showed honeycomb-like network structures (Figure 3C) and fragmented pore structures (Figure 3D), respectively. According to the results of compressive tests, 3GAs (small GO sheets) showed small compressive stress and elastic deformation during the compressive tests. The elasticity, compressive stress, and Young's modulus of 3GAs produced by large GO sheets were significantly increased compared to the $3 \mathrm{GAs}$ produced by small GO sheets (Figure 3E) because large GO sheets can be assembled into a porous structure with a large $\pi-\pi$ stacking area and fewer dislocations. Wu et al. also used the large GO sheets $(20-50 \mu \mathrm{m})$ (Figure 3F) to prepare the 3GAs with superelasticity of up to $98 \%$ strain and excellent fatigue resistance (maintain structural integrity after 1,000 compression cycles) (Figure 3G) (Wu et al., 2015). Therefore, the use of large GO sheets is beneficial for the preparation of $3 \mathrm{GAs}$ with excellent mechanical properties.

\section{Interactions Between Graphene Sheets}

Due to the graphene sheet stacks to construct the graphene network, it is an effective method to obtain robust 3GAs by enhancing the interactions between graphene sheets. Guo et al. fabricated binary carbon porous materials by the ink-printing solution of GO and multi-walled carbon nanotubes (MWNTs), followed by reduction and confined processes (Figure 4A) (Guo et al., 2018). Thanks to the hierarchical structures and synergistic 

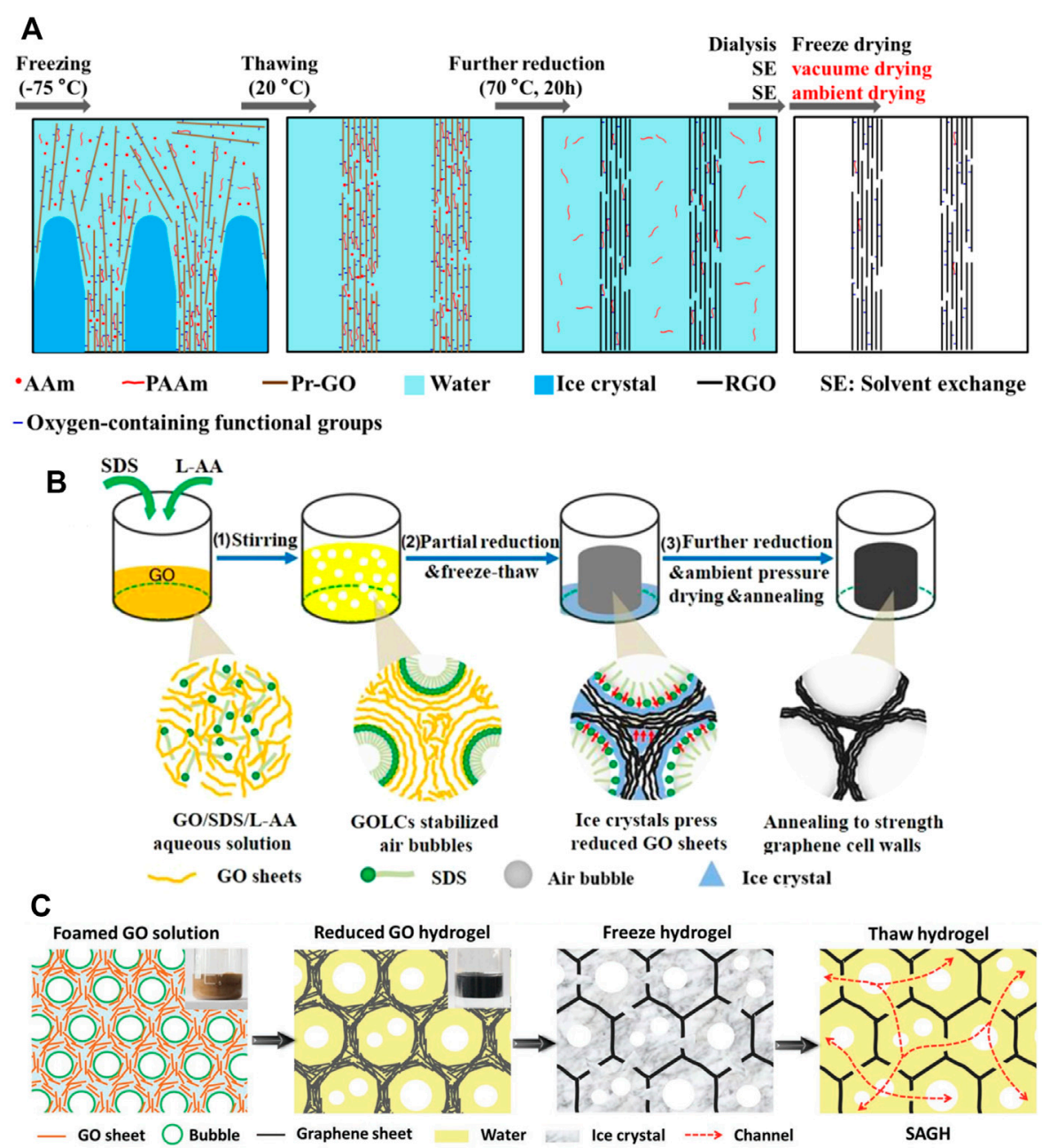

FIGURE 2 | (A) Schematic model for the formation of the 3GA through in situ polymerization of Aam (Li et al., 2016). (B) Illustration of the preparation process of the 3GA by using GO liquid crystal-stabilized bubbles as a template (Zhang et al., 2019). (C) Schematic illustration of the air-dryable graphene hydrogel by using ice crystals and air bubbles as templates (Yang et al., 2019).

effect of graphene and MWNTs, the binary carbon porous materials showed recoverable stretching elasticity (up to $100 \%$ strain) and excellent fatigue resistance (100,000 cycles) (Figure 4B). Huang et al. prepared two kinds of crosslinked GO porous materials by using $\mathrm{La}^{3+}$ and polyethylenimine (PEI) as crosslinkers, respectively (Huang et al., 2013b). The $\mathrm{La}^{3+}$ $\left(55 \mathrm{mg} \mathrm{cm}^{-3}\right)$ (Figure 4C) and PEI- $\left(60 \mathrm{mg} \mathrm{cm}^{-3}\right)$ (Figure 4D) crosslinked porous materials showed a Young's modulus of 10 and $20 \mathrm{MPa}$ and the compressive stress of 10 and $12 \mathrm{MPa}$, respectively. Due to the crosslinking of $\mathrm{La}^{3+}$ and PEI, the mechanical properties of crosslinked GO porous materials are much better than those without crosslinking. Based on the 3GAs prepared in our previous work ( $\mathrm{Li}$ et al., 2016), we fabricated the 3GAs that could withstand extreme stress and strain through a simple thermal treatment (Li et al., 2018b). After the thermal treatment $\left(400-1,000^{\circ} \mathrm{C}\right)$, most of oxygen-containing functional groups on the graphene sheets were eliminated, and $\pi-\pi$ interaction between the graphene sheets were enhanced, forming an ordered stacked graphene sheets in cell walls (Figure 4E). The 3GA could recover its own shape and network structures after the extreme compression test (100, $000 \mathrm{~N}$ ) of $60 \mathrm{~min}$ (Figure 4F). The 3GA exhibited the compressive stress of approximately 1,000 MPa and a strain of $99.8 \%$, which are higher than those of other elastic porous materials.

\section{Graphene Network Structures}

Because of the lack of effective control methods, GO sheets tend to assemble into a disordered graphene porous structure. These 3GAs with disordered network structures generally exhibit brittle 

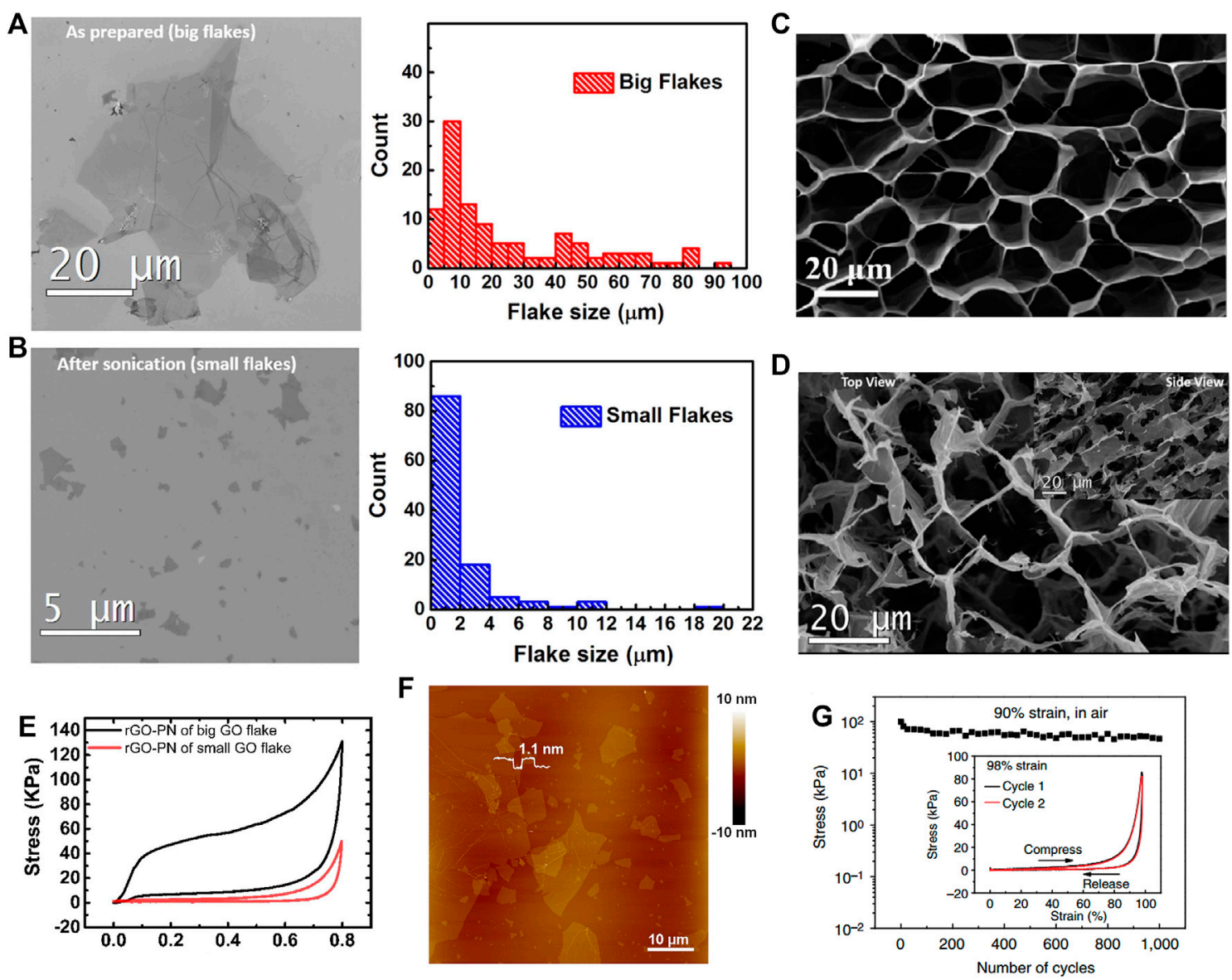

FIGURE 3 | SEM images and size distributions of large (A) and small (B) GO sheets (Ni et al., 2015). SEM images of $3 G A s$ prepared by large (C) and small (D) GO sheets (Ni et al., 2015). (E) Compressive stress-strain curves of 3GAs prepared by large and small GO sheets (Ni et al., 2015). (F) AFM image of GO sheets with a large size of 20-50 $\mu \mathrm{m}$ (Wu et al., 2015). (G) Compressive stress-strain curves of 3GA for 1,000 cycles in air. Inset: Compressive stress-strain curves of 3GA for two cycles under the compressive strain of $98 \%$ (Wu et al., 2015).

and weak mechanical properties. How to construct ordered graphene porous structures is one of the key factors for preparing 3GAs with excellent mechanical properties. Recently, Qiu et al. used a simple method to fabricate elastic 3GAs with biomimetic hierarchical structures (Figure 5A) (Qiu et al., 2012). The ascorbic acid (reducing agent) was added to the GO solution, followed by a partial reduction $\left(100^{\circ} \mathrm{C}, 30 \mathrm{~min}\right)$ and directional freezing $\left(-78^{\circ} \mathrm{C}, 30 \mathrm{~min}\right)$. The partially reduced GO sheets were forced to align along the oriented ice crystals, resulting in the formation of ordered 3D network structures which were determined by ice templates. The weak hydrogel was strengthened due to the enhancement of $\pi-\pi$ attraction during further reduction at $100{ }^{\circ} \mathrm{C}$ for $8 \mathrm{~h}$. After freeze drying, 3GAs showed a honeycomb-like cell structures (Figure 5B) with cell walls composed of oriented graphene sheets (Figure 5C). The 3GA with a low density $\left(5.1 \mathrm{mg} \mathrm{cm}^{-3}\right)$ can withstand more than 50,000 times its weight under compression of $80 \%$ strain and retain its network structure intact, which was completely different from other 3GAs with random porous structures (Figure 5D). Meanwhile, the honeycomb-like cell structures and face-to-face stacked graphene sheets of the cell wall provide the excellent paths for electron transport, resulting in the high electrical property of 3GAs. The electrical conductivity of 3GAs is three times higher than that of the carbon-based porous material with a similar density (Figure 5E).

Researchers used other strategies to prepare robust 3GAs with ordered porous structures. Peng et al. used an ink-printing technique to construct ultralight and elastic 3GAs with biomimetic hierarchical structures (Figure 5F) (Peng et al., 2019). The macroscopic hollow scaffold and the microscopic honeycomb biomimetic-layered structure are realized by partially reducing the GO ink and combining the directional freezing process. The resulting 3GAs show exceptional resilience and a density of $8.5 \mathrm{mg} \mathrm{cm}^{-3}$. The $3 \mathrm{GA}$ can 

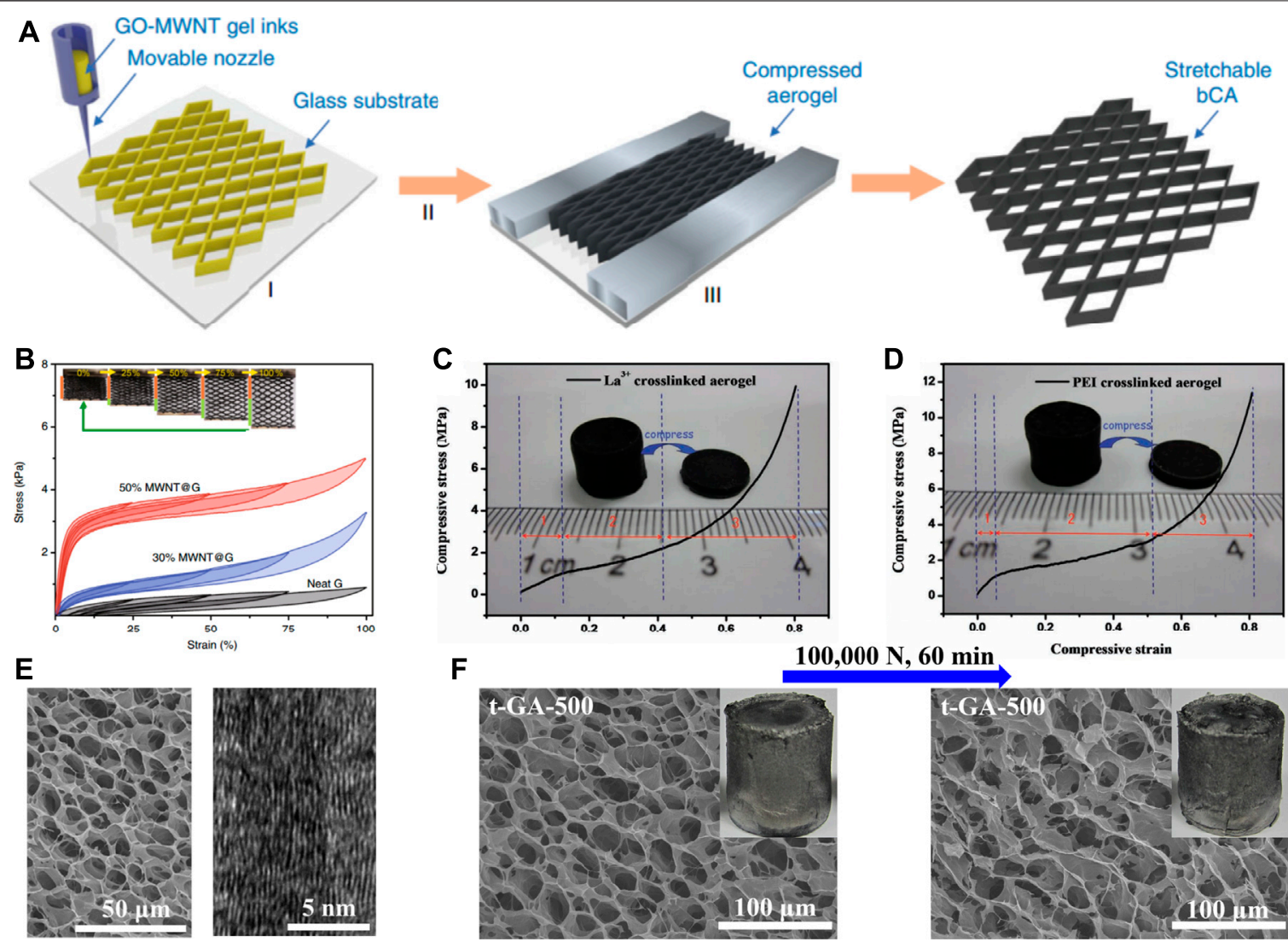

FIGURE 4 | (A) Schematic illustration of the hierarchical synergistic assembly for fabrication of binary carbon porous material through the 3D printing technique (Guo et al., 2018). (B) Stress-strain curves of 3GAs with 25, 50, 75, and 100\% tensile strains (Guo et al., 2018). Compressive stress-strain curves of the La ${ }^{3+}$ - (C) and PEI- (D) crosslinked GO porous materials (Guo et al., 2018). (E) SEM image and HRTEM image of 3GAs (Li et al., 2018b). (F) SEM image and digital image of 3GAs before and after compression under 100, $000 \mathrm{~N}$ for $60 \mathrm{~min}$ (Li et al., 2018b).

maintain its height of $90.1 \%$ after 10 cycles of $95 \%$ strain compression under the maximum pressure of $0.066 \mathrm{MPa}$. Yeo et al. used a strategy to prepare an ultralight and superelastic 3GAs with closed cellular structures (Figure 5G) (Yeo et al., 2018). First, spherical solid-shelled bubbles were prepared by controlled synthesis of functionalized GO sheets. Then, bubbles were assembled into a 3D network, resulting in the 3GAs with ordered rhombic dodecahedral structures through posttreatment. Benefiting from the ordered closed-cellular network for stress dissipation, the $3 \mathrm{GA}$ of $7.7 \mathrm{mg} \mathrm{cm}^{-3}$ exhibited the Young's modulus of $0.3 \mathrm{MPa}$ and retained the elasticity after the compression of $87 \%$ strain. Recently, we used melamine foam (MF) as the sacrificial skeleton to prepare the superelastic and durable 3GAs (Li et al., 2018c). The skeleton of MF effectively prevented serious accumulation of GO sheets, resulting in the arbitrary-shaped 3GAs with ordered network structures (Figure 5H). The 3GA with the low density $\left(4.2 \mathrm{mg} \mathrm{cm}^{-3}\right)$ showed the high elasticity with high compressive stress $(0.556 \mathrm{MPa})$ and high compressive strain (95\%) (Figure 5I).

\section{DISCUSSION AND PERSPECTIVES}

The great potential of elastic and robust 3GAs in a variety of applications has drawn great attention although much process has been achieved on the simple preparation and functionalization of 3GAs. However, there are still some difficulties to overcome. First, most graphene gels have a fragile network structure and cannot withstand the capillary forces during air drying, resulting in structural collapse. Previous methods mainly connected graphene sheets via weak bonds (van der Waals force and ionic bond) to establish graphene networks. Therefore, it is necessary to develop an effective method to introduce more covalent bonds between graphene sheets in order to increase the stress between graphene sheets, forming the robust graphene network structure. Second, in order to obtain intact 3GAs by air drying, it is usually necessary to undergo a long-term solvent replacement and drying process, thereby increasing production costs. How to reduce the preparation cost of large-scale air drying? This requires the removal of time-consuming steps (such as 


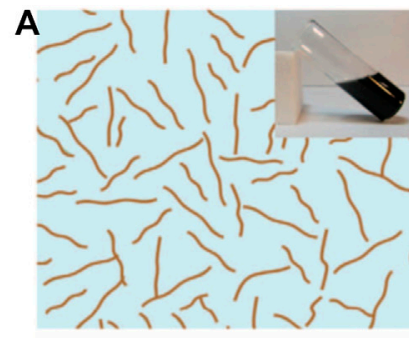

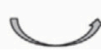

Freezing

B

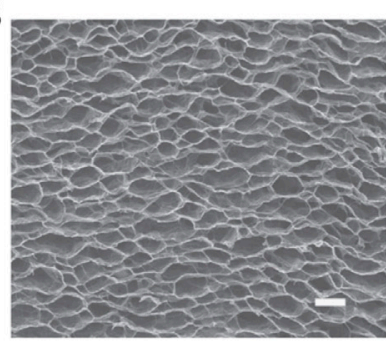

F

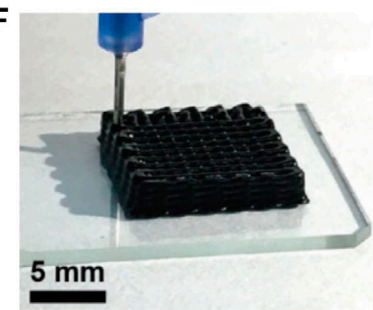

G

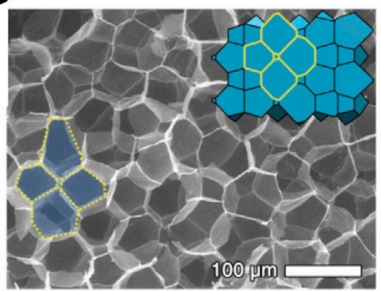

H c

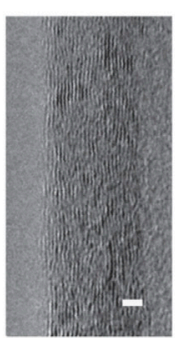

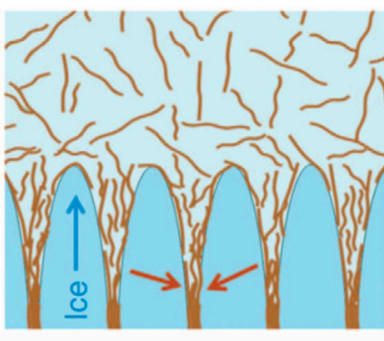

Thawing

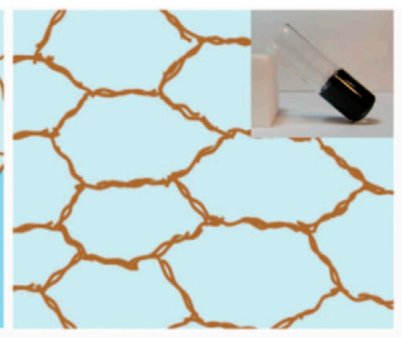

12

Further reduction and freeze drying
D
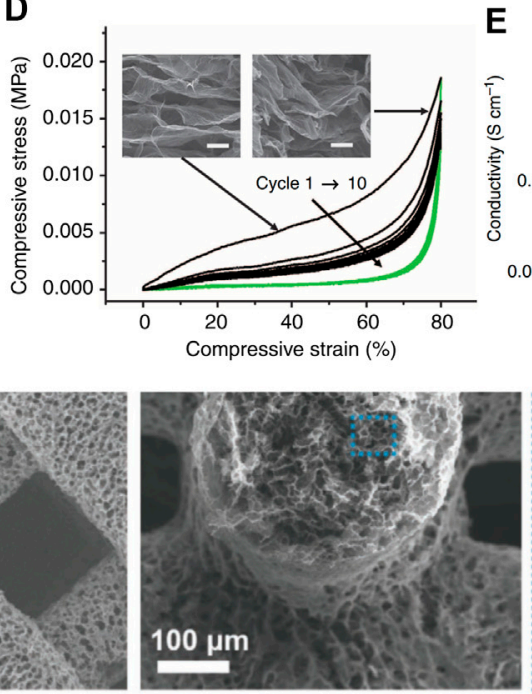
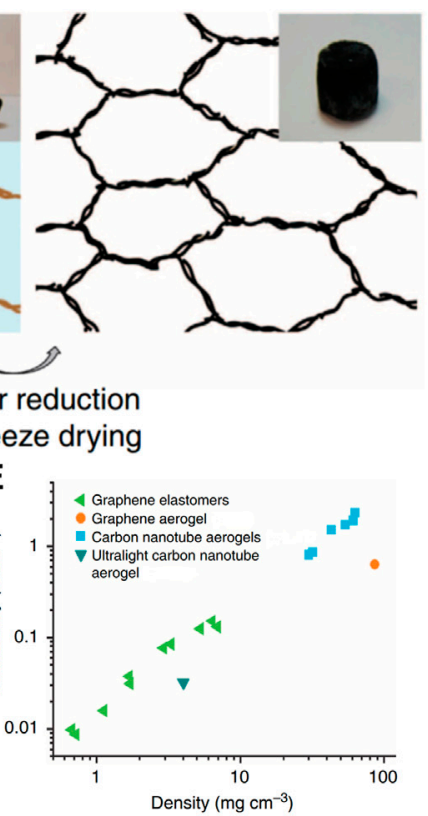
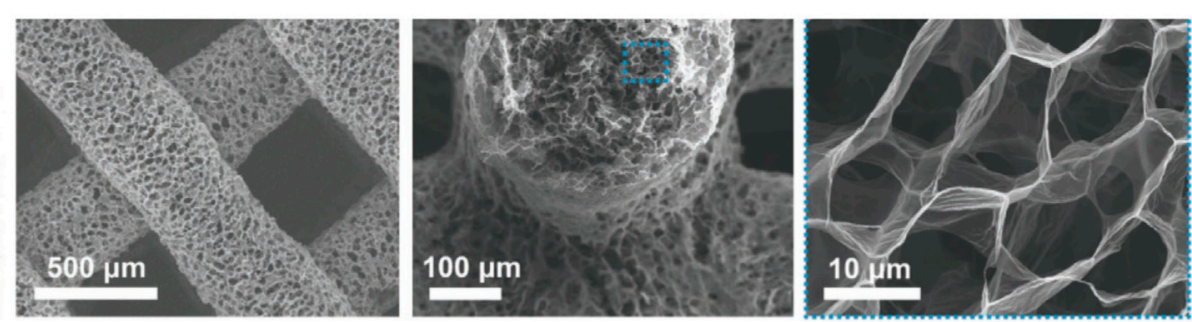

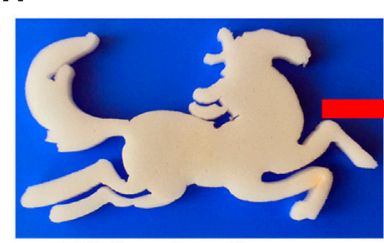

Melamine foam

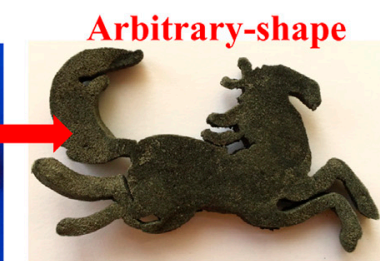

Graphene aerogel

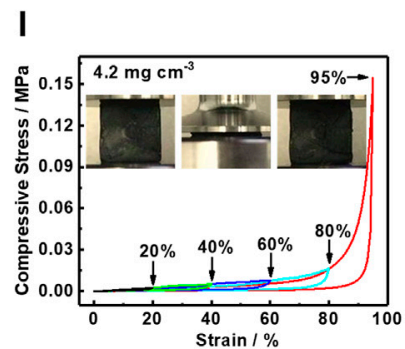

FIGURE 5 | (A) Schematic illustrations for the fabrication of 3GA with ordered porous structure by using a two-step strategy (Qiu et al., 2012). SEM image (B) and HRTEM image (C) of 3GA (Qiu et al., 2012). (D) Compressive stress-strain curves of 10 cycles. Inset: SEM images of 3GA under compression during a cycle (Qiu et al., 2012). (E) The electrical conductivity of 3GAs was compared with those of other carbon-based porous materials as a function of density (Qiu et al., 2012). (F) Digital and SEM images of 3GAs prepared by the 3D printing technology (Peng et al., 2019). (G) SEM image of 3GA (Peng et al., 2019). (H) Fabrication of arbitrary-shaped 3GA by using melamine foam as the sacrificial skeleton (Li et al., 2018c). (I) Stress-strain curves of 3GA at a maximum compressive strain of 95\% (Li et al., 2018c).

replacement of water in the graphene hydrogel to a solvent having a small surface tension) and raising the temperature during air drying to increase the drying efficiency. Therefore, it is necessary to develop a new graphene hydrogel system that can withstand the damage of the graphene network by the capillary force caused by water evaporation at a high temperature. Third, in order to expand the application of 3GAs in electrocatalysis, energy storage, and electromagnetic shielding, it is also important to prepare 3GAs with mesoporous and microporous structures by using the air drying method. How to prepare the $3 \mathrm{GAs}$ with mesoporous and microporous structures and keep the mesoporous and microporous structure intact after air drying, which puts higher requirements on the design of the graphene hydrogel system. Therefore, continuous innovation and research and development are needed to further enhance the structure of 
the 3GA network, reduce the large-scale preparation cost of 3GAs, and expand the application range of $3 \mathrm{GAs}$.

\section{AUTHOR CONTRIBUTIONS}

MD and CL conceived the concept of the review. MD drafted the manuscript, searched for updated bibliography, and prepared the figures. MD and CL revised, corrected, and edited the manuscript.

\section{REFERENCE}

Bi, H., Chen, I.-W., Lin, T., and Huang, F. (2015). A New Tubular Graphene Form of a Tetrahedrally Connected Cellular Structure. Adv. Mater. 27, 5943-5949. doi:10.1002/adma.201502682

Bi, H., Xie, X., Yin, K., Zhou, Y., Wan, S., He, L., et al. (2012). Spongy Graphene as a Highly Efficient and Recyclable Sorbent for Oils and Organic Solvents. Adv. Funct. Mater. 22, 4421-4425. doi:10.1002/adfm.201200888

Cao, X., YinZhang, Z. H., and Zhang, H. (2014). Three-dimensional Graphene Materials: Preparation, Structures and Application in Supercapacitors. Energy Environ. Sci. 7, 1850-1865. doi:10.1039/c4ee00050a

Gallé, C. (2001). Effect of Drying on Cement-Based Materials Pore Structure as Identified by Mercury Intrusion Porosimetry. Cement Concrete Res. 31, 1467-1477. doi:10.1016/s0008-8846(01)00594-4

Guo, F., Jiang, Y., Xu, Z., Xiao, Y., Fang, B., Liu, Y., et al. (2018). Highly Stretchable Carbon Aerogels. Nat. Commun. 9, 881. doi:10.1038/s41467-018-03268-y

Hu, H., Zhao, Z., Wan, W., Gogotsi, Y., and Qiu, J. (2013). Ultralight and Highly Compressible Graphene Aerogels. Adv. Mater. 25, 2219-2223. doi:10.1002/ adma.201204530

Huang, H., Chen, P., Zhang, X., Lu, Y., and Zhan, W. (2013). Edge-to-edge Assembled Graphene Oxide Aerogels with Outstanding Mechanical Performance and Superhigh Chemical Activity. Small 9 (8), 1397-1404. doi:10.1002/smll.201202965

Huang, H., Chen, P., Zhang, X., Lu, Y., and Zhan, W. (2013). Edge-to-Edge Assembled Graphene Oxide Aerogels with Outstanding Mechanical Performance and Superhigh Chemical Activity. Small 9, 1397-1404. doi:10. 1002/smll.201202965

Huo, B., Jiang, D., Cao, X., Liang, H., Liu, Z., Li, C., et al. (2019). N-doped Graphene /carbon Hybrid Aerogels for Efficient Solar Steam Generation. Carbon 142, 13-19. doi:10.1016/j.carbon.2018.10.008

Jiang, D., Li, C., Yang, W., Zhang, J., and Liu, J. (2017). Fabrication of an Arbitrary-Shaped and Nitrogen-Doped Graphene Aerogel for Highly Compressible All Solid-State Supercapacitors. J. Mater. Chem. A. 5, 18684-18690. doi:10.1039/c7ta04917j

Kistler, S. (1931). Coherent Expanded Aerogels. J. Phys. Chem. 36, 52-64.

Kistler, S. S. (1931). Coherent Expanded Aerogels and Jellies. Nature 127, 741. doi:10.1038/127741a0

Kong, H., Song, Z., Li, W., Bao, Y., Qu, D., Ma, Y., et al. (2021). Skin-inspired HairEpidermis-Dermis Hierarchical Structures for Electronic Skin Sensors with High Sensitivity over a Wide Linear Range. ACS Nano 15 (10), 16218-16227. doi:10.1021/acsnano.1c05199

Kong, W., Kum, H., Bae, S.-H., Shim, J., Kim, H., Kong, L., et al. (2019). Path towards Graphene Commercialization from Lab to Market. Nat. Nanotechnol. 14, 927-938. doi:10.1038/s41565-019-0555-2

Li, C., Ding, M., Zhang, B., Qiao, X., and Liu, C.-Y. (2018). Graphene Aerogels that Withstand Extreme Compressive Stress and Strain. Nanoscale 10, 18291-18299. doi:10.1039/c8nr04824j

Li, C., Jiang, D., Huo, B., Ding, M., Huang, C., Jia, D., et al. (2019). Scalable and Robust Bilayer Polymer Foams for Highly Efficient and Stable Solar Desalination. Nano Energy 60, 841-849. doi:10.1016/j.nanoen.2019.03.087

Li, C., Qiu, L., Zhang, B., Li, D., and Liu, C.-Y. (2016). Robust Vacuum-/air-Dried Graphene Aerogels and Fast Recoverable Shape-Memory Hybrid Foams. Adv. Mater. 28, 1510-1516. doi:10.1002/adma.201504317

Li, C., and Shi, G. (2012). Three-dimensional Graphene Architectures. Nanoscale 4, 5549-5563. doi:10.1039/c2nr31467c

\section{FUNDING}

This work was supported by the National Natural Science Foundation of China (51802168), the China Postdoctoral Science Foundation (2018M630753), the Natural Science Foundation of Shandong Province (ZR2018BEM006), the Applied Basic Research Fund of Qingdao (No.19-6-2-76cg), and the Qingdao Postdoctoral Application Research Project.

Li, C. W., Jiang, D. G., Liang, H., Huo, B. B., Liu, C. Y., Yang, W. R., et al. (2018). Superelastic and Arbitrary-Shaped Graphene Aerogels with Sacrificial Skeleton of Melamine Foam for Varied Applications. Adv. Funct. Mater. 28, 170467. doi:10.1002/adfm.201704674

Li, H., Jia, D., Ding, M., Zhou, L., Wang, K., Liu, J., et al. (2021). Robust 3D Graphene/ Cellulose Nanocrystals Hybrid Lamella Network for Stable and Highly Efficient Solar Desalination. Sol. RRL 5, 2100317. doi:10.1002/solr.202100317

Li, H., Tao, Y., Zhang, C., Liu, D., Luo, J., Fan, W., et al. (2018). Dense Graphene Monolith for High Volumetric Energy Density Li-S Batteries. Adv. Energ. Mater. 8 (18), 1703438. doi:10.1002/aenm.201703438

Li, Y., Chen, J., Huang, L., Li, C., Hong, J.-D., and Shi, G. (2014). Highly Compressible Macroporous Graphene Monoliths via an Improved Hydrothermal Process. Adv. Mater. 26, 4789-4793. doi:10.1002/adma.201400657

Liang, H., Jiang, D., Wei, S., Cao, X., Chen, T., Huo, B., et al. (2018). 3D Cellular CoS1.097/nitrogen Doped Graphene Foam: a Durable and Self-Supported Bifunctional Electrode for Overall Water Splitting. J. Mater. Chem. A. 6, 16235-16245. doi:10.1039/c8ta05407j

Lu, H., Li, C., Zhang, B., Qiao, X., and Liu, C.-Y. (2016). Toward Highly Compressible Graphene Aerogels of Enhanced Mechanical Performance with Polymer. RSC Adv. 6, 43007-43015. doi:10.1039/c6ra04995h

Ma, Y., and Chen, Y. (2015). Three-dimensional Graphene Networks: Synthesis, Properties and Applications. Natl. Sci. Rev. 2, 40-53. doi:10. 1093/nsr/nwu072

Maleki, H., Durães, L., and Portugal, A. (2015). Development of Mechanically strong Ambient Pressure Dried Silica Aerogels with Optimized Properties. J. Phys. Chem. C 119, 7689-7703. doi:10.1021/jp5116004

Maleki, H. (2016). Recent Advances in Aerogels for Environmental Remediation Applications: A Review. Chem. Eng. J. 300, 98-118. doi:10.1016/j.cej.2016. 04.098

Nardecchia, S., Carriazo, D., Ferrer, M. L., Gutiérrez, M. C., and del Monte, F. (2013). Three Dimensional Macroporous Architectures and Aerogels Built of Carbon Nanotubes And/or Graphene: Synthesis and Applications. Chem. Soc. Rev. 42, 794-830. doi:10.1039/c2cs35353a

Ni, N., Barg, S., Garcia-Tunon, E., Macul Perez, F., Miranda, M., Lu, C., et al. (2015). Understanding Mechanical Response of Elastomeric Graphene Networks. Sci. Rep. 5, 13712. doi:10.1038/srep13712

Novoselov, K. S., Geim, A. K., Morozov, S. V., Jiang, D., Katsnelson, M. I., Grigorieva, I. V., et al. (2005). Two-dimensional Gas of Massless Dirac Fermions in Graphene. Nature 438, 197-200. doi:10.1038/nature04233

Peng, M., Wen, Z., Xie, L., Cheng, J., Jia, Z., Shi, D., et al. (2019). 3D Printing of Ultralight Biomimetic Hierarchical Graphene Materials with Exceptional Stiffness and Resilience. Adv. Mater. 31, 1902930. doi:10.1002/adma.201902930

Qiu, L., Bulut Coskun, M., Tang, Y., Liu, J. Z., Alan, T., Ding, J., et al. (2016). Ultrafast Dynamic Piezoresistive Response of Graphene-Based Cellular Elastomers. Adv. Mater. 28, 194-200. doi:10.1002/adma.201503957

Qiu, L., He, Z., and Li, D. (2018). Multifunctional Cellular Materials Based on 2d Nanomaterials: Prospects and Challenges. Adv. Mater. 30, 1704850. doi:10. 1002/adma.201704850

Qiu, L., Liu, D., Wang, Y., Cheng, C., Zhou, K., Ding, J., et al. (2014). Mechanically Robust, Electrically Conductive and Stimuli-Responsive Binary Network Hydrogels Enabled by Superelastic Graphene Aerogels. Adv. Mater. 26, 3333-3337. doi:10.1002/adma.201305359

Qiu, L., Liu, J. Z., Chang, S. L. Y., Wu, Y., and Li, D. (2012). Biomimetic Superelastic Graphene-Based Cellular Monoliths. Nat. Commun. 3, 1241. doi:10.1038/ ncomms 2251 
Qu, F., Jiang, D., Liu, Z., Zhang, A., Zheng, Y., Jia, Q., et al. (2020). Hierarchical Polypyrrole/graphene/melamine Composite Foam for Highly Compressible All-Solid-State Supercapacitors. Electrochimica Acta 353, 136600. doi:10. 1016/j.electacta.2020.136600

Scherer, G. W. (1993). Freezing Gels. J. Non-Crystalline Sol. 155, 1-25. doi:10.1016/ 0022-3093(93)90467-c

Smith, D. M., Scherer, G. W., and Anderson, J. M. (1995). Shrinkage during Drying of Silica Gel. J. Non-Crystalline Sol. 188, 191-206. doi:10.1016/0022-3093(95) 00187-5

Sui, Z., Meng, Q., Zhang, X., Ma, R., and Cao, B. (2012). Green Synthesis of Carbon Nanotube-Graphene Hybrid Aerogels and Their Use as Versatile Agents for Water Purification. J. Mater. Chem. 22, 8767-8771. doi:10.1039/c2jm00055e

Sun, H., Xu, Z., and Gao, C. (2013). Multifunctional, Ultra-flyweight, Synergistically Assembled Carbon Aerogels. Adv. Mater. 25, 2554-2560. doi:10.1002/adma.201204576

Wang, Z., Dai, Z., Wu, J., Zhao, N., and Xu, J. (2013). Vacuum-dried Robust Bridged Silsesquioxane Aerogels. Adv. Mater. 25, 4494-4497. doi:10.1002/adma.201301617

Wu, Y., Yi, N., Huang, L., Zhang, T., Fang, S., Chang, H., et al. (2015). Three-dimensionally Bonded Spongy Graphene Material with Super Compressive Elasticity and Near-Zero Poisson's Ratio. Nat. Commun. 6, 6141. doi:10.1038/ncomms7141

Yang, H., Li, Z., Sun, G., Jin, X., Jin, B., Zhang, P., et al. (2019). Superplastic AirDryable Graphene Hydrogels for Wet-Press Assembly of Ultrastrong Superelastic Aerogels with Infinite Macroscale. Adv. Funct. Mater. 29, 1901917. doi:10.1002/adfm.201901917

Yang, Y., Zhao, R., Zhang, T., Zhao, K., Xiao, P., Ma, Y., et al. (2018). Graphenebased Standalone Solar Energy Converter for Water Desalination and Purification. ACS Nano 12 (1), 829-835. doi:10.1021/acsnano.7b08196

Yeo, S. J., Oh, M. J., Jun, H. M., Lee, M., Bae, J. G., Kim, Y., et al. (2018). A Plesiohedral Cellular Network of Graphene Bubbles for Ultralight, strong, and Superelastic Materials. Adv. Mater. 30, 1802997. doi:10.1002/adma.201802997

Zhang, P., Li, J., Lv, L., Zhao, Y., and Qu, L. (2017). Vertically Aligned Graphene Sheets Membrane for Highly Efficient Solar thermal Generation of Clean Water. ACS Nano 11, 5087-5093. doi:10.1021/acsnano.7b01965
Zhang, X., Sui, Z., Xu, B., Yue, S., Luo, Y., Zhan, W., et al. (2011). Mechanically strong and Highly Conductive Graphene Aerogel and its Use as Electrodes for Electrochemical Power Sources. J. Mater. Chem. 21, 6494-6497. doi:10.1039/ cljm10239g

Zhang, X., Zhang, T., Wang, Z., Ren, Z., Yan, S., Duan, Y., et al. (2019). Ultralight, Superelastic, and Fatigue-Resistant Graphene Aerogel Templated by Graphene Oxide Liquid crystal Stabilized Air Bubbles. ACS Appl. Mater. Inter. 11, 1303-1310. doi:10.1021/acsami.8b18606

Zhang, Z., and Scherer, G. W. (2017). Supercritical Drying of Cementitious Materials. Cement Concrete Res. 99, 137-154. doi:10.1016/j.cemconres.2017. 05.005

Zhao, J., Liu, L., and Li, F. (2015). Graphene Oxide: Physics and Applications. Springer Berlin, Germany.

Zhu, C., Han, T. Y.-J., Duoss, E. B., Golobic, A. M., Kuntz, J. D., Spadaccini, C. M., et al. (2015). Highly Compressible 3D Periodic Graphene Aerogel Microlattices. Nat. Commun. 6, 6962. doi:10.1038/ncomms7962

Conflict of Interest: The authors declare that the research was conducted in the absence of any commercial or financial relationships that could be construed as a potential conflict of interest.

Publisher's Note: All claims expressed in this article are solely those of the authors and do not necessarily represent those of their affiliated organizations, or those of the publisher, the editors, and the reviewers. Any product that may be evaluated in this article, or claim that may be made by its manufacturer, is not guaranteed or endorsed by the publisher.

Copyright (C) 2022 Ding and Li. This is an open-access article distributed under the terms of the Creative Commons Attribution License (CC BY). The use, distribution or reproduction in other forums is permitted, provided the original author $(s)$ and the copyright owner(s) are credited and that the original publication in this journal is cited, in accordance with accepted academic practice. No use, distribution or reproduction is permitted which does not comply with these terms. 